\title{
Cooperative Diversity over Log-Normal Fading Channels: Performance Analysis and Optimization
}

\author{
Majid Safari, Student Member, IEEE, and Murat Uysal, Senior Member, IEEE
}

\begin{abstract}
Although there has been a growing interest on cooperative diversity, the current literature is mainly limited to the results obtained for Rayleigh, Rician, or Nakagami fading channels. In this paper, we investigate the performance of cooperative diversity schemes over log-normal fading channels which provide an accurate channel model for indoor wireless environments. We focus on single-relay cooperative networks with amplify-and-forward relaying and consider three TDMA-based cooperation protocols which correspond to distributed implementations of MIMO (multi-input multi-output), SIMO (single-input multi-output), and MISO (multi-input single-output) schemes. For each protocol under consideration, we derive upper bounds on pairwise error probability over log-normal channels and quantify the diversity advantages. Based on the minimization of a union bound on the bit error rate performance, we further formulate optimal power allocation schemes which demonstrate significant performance gains over their counterparts with equal power allocation.
\end{abstract}

Index Terms-Cooperative diversity, distributed space-time codes, pairwise error probability, power allocation, log-normal fading.

\section{INTRODUCTION}

A major impairment in wireless channels is the multipathinduced fading which causes random fluctuations in the received signal level. For a typical mobile wireless channel in urban areas where there is no line of sight propagation and the number of scatters is considerably large, the application of central limit theory indicates that the complex fading channel coefficient can be modeled with two quadrature components which are zero-mean Gaussian random processes. As a result, the amplitude of the fading envelope follows a Rayleigh distribution. In terms of error rate performance, Rayleigh fading converts the exponential dependency of the bit error rate on the signal-to-noise ratio (SNR) for the classical additive white Gaussian noise (AWGN) channel into an approximately inverse linear one, resulting in a large SNR penalty.

Diversity techniques are widely adopted in wireless communication systems as a counter-measure to fading effects providing redundancy across independently faded diversity branches. Deployment of multiple transmit and/or receive antennas realizes the advantages of spatial diversity. Through the use of suitably designed space-time codes [1]-[3], diversity

Manuscript received April 12, 2007; revised October 19, 2007; accepted November 9, 2007. The associate editor coordinating the review of this paper and approving it for publication was J. Andrews. This paper was presented in part at the 10th Canadian Workshop on Information Theory (CWIT07), Edmonton, Alberta, Canada, June 2007. The work of M. Uysal is supported in part by a Natural Sciences and Engineering Research Council of Canada (NSERC) Special Research Opportunity Grant (SROPJ305821-05).

The authors are with the Department of Electrical and Computer Engineering, University of Waterloo, Waterloo, ON, N2L3G1, Canada (e-mail: m3safari@uwaterloo.ca, muysal@ece.uwaterloo.ca).

Digital Object Identifier 10.1109/TWC.2008.070393. and/or multiplexing gains can be achieved at no cost in terms of transmission time and bandwidth expansion.

An alternative form of spatial diversity, referred as "cooperative diversity" [4]-[9], has been recently proposed to realize diversity advantages in a distributed manner. In this technique, spatial diversity gain is extracted by creating virtual antenna arrays through cooperating users. In their pioneering work [6], Laneman et al. have proposed a two-phase cooperation protocol which is able to extract the full spatial diversity. In the first phase (i.e., broadcasting phase), the source transmits to the destination and relay terminals. In the second phase (i.e., relaying phase), the relays transmit their received signals to the destination using either orthogonal sub-channels (repetition based cooperative diversity) or the same subchannel (space-time coded cooperative diversity). The receiver coherently combines the received signals over two phase durations realizing receive diversity in a distributed manner. In [8], Nabar et al. have established a unified framework for user cooperation protocols in single-relay wireless networks. They propose three TDMA (time division multiple access)based cooperation protocols so-called Protocol I, Protocol II, and Protocol III which realize distributed MIMO (multi-input multi-output), SIMO (single-input multi-output) and MISO (multi-input single-output) configurations, respectively. Protocol II coincides with Laneman et.al.'s cooperation protocol proposed in [6]. Protocol I differs from Protocol II in the sense that the source terminal continues transmission in the relaying phase and it has been recently shown in [10] that this protocol is optimal in terms of diversity-multiplexing trade-off.

Although there has been a growing interest on cooperative diversity, the current literature is mainly limited to the results obtained for Rayleigh, Rician, or Nakagami fading channels. These channel models accurately capture the characteristics of outdoor wireless channels, however log-normal fading is found to be a better fit for indoor radio propagation environments [11]-[14]. It has been demonstrated through empirical fading channel measurements that short-term and long-term fading effects tend to get mixed in indoor wireless channels and the log-normal statistics tend to dominate, and therefore, describe the distribution of the channel coefficient. The performance of receive antenna diversity in log-normal fading channels has been studied in [15], [16]. In [15], Alouini and Simon have derived outage probability and amount of fading for maximalratio combining, selection combining, and switch-and-stay combining schemes assuming the deployment of two receive antennas. In [16], Piboongungon and Aalo have derived the outage probability for selection combining with more than two receive antennas.

To the best of our knowledge, there have been no previous 
results reported on cooperative diversity in the presence of lognormal fading. Aiming to fill this research gap, the current paper analyzes the error rate performance of cooperative diversity schemes over log-normal fading channels through the derivation of upper bounds on pairwise error probability (PEP) expressions and further optimizes the performance through power allocation among cooperating nodes taking into account the relay location. Specifically, we consider single-relay cooperative networks under the framework of Nabar et al.'s [8] cooperation protocols which effectively cover distributed MIMO, SIMO, and MISO configurations. Our performance analysis builds upon the derivation of Chernoff bounds on PEP and a union bound on the bit error rate (BER) performance. Since conventional definition of diversity order is of no use for log-normal channels, we define so-called "relative diversity order (RDO)" to quantify the diversity advantages and to compare the performance of various competing schemes. We further formulate a power allocation scheme for cooperative diversity schemes under consideration and optimize their BER performance over log-normal channels. The optimized protocols demonstrate significant performance gains over their counterparts with equal power allocation.

The rest of the paper is organized as follows: In Section II, we introduce the relay-assisted transmission model and describe the received signal models for Protocols I, II, and III. In Section III, we derive upper bounds on the PEP for each protocol over log-normal fading channels. In Section IV, we investigate optimal power allocation with an objective function given in terms of a union bound on the BER. In Section V, we analyze the diversity gains for each of the protocols under consideration with both equal and optimal power allocation. In Section VI, we present an extensive Monte-Carlo simulation study to confirm the diversity gains predicted by the derived analytical expressions and to compare them with several benchmark schemes. Section VII finally concludes the paper.

\section{SyStem MODEL}

We consider a single-relay scenario where terminals operate in half-duplex mode and are equipped with single transmit and receive antennas. The fading coefficients over sourceto-destination $(\mathrm{S} \rightarrow \mathrm{D})$, source-to-relay $(\mathrm{S} \rightarrow \mathrm{R})$, and relay-todestination $(\mathrm{R} \rightarrow \mathrm{D})$ links are denoted by $h_{S D}, h_{S R}$, and $h_{R D}$ which are assumed to be independent and identically distributed (i.i.d) log-normal random variables. Dropping indexes for convenience, the probability density function (pdf) of $h$ is given by [15]

$$
f_{h}(h)=\frac{\xi}{\sqrt{2 \pi} \sigma_{a} h} \exp \left[-\frac{\left(20 \log _{10} h-m_{a}\right)^{2}}{2 \sigma_{a}^{2}}\right]
$$

where $m_{a}$ and $\sigma_{a}^{2}$ are, respectively, the mean and variance of $a \stackrel{\text { def }}{=} 20 \log _{10} h$ which follows Gaussian distribution and $\xi=20 / \ln 10=8.6859$. To ensure that the fading does not attenuate or amplify the average power, $h$ is normalized such that $E\left[h^{2}\right]=1$. Doing so requires

$$
E\left[h^{2}\right]=E\left[10^{2 a / 20}\right]=E\left[e^{2 a / \xi}\right]=M_{a}(2 / \xi)=1
$$

where $M_{a}(s)$ is the moment generating function of $a$ and is given by $M_{a}(s)=\exp \left(m_{a} s+\sigma_{a}^{2} s^{2} / 2\right)$ [17]. Hence, choosing $m_{a}=-\sigma_{a}^{2} / \xi$ makes the average power loss due to channel fading unity. Typical values of the standard deviation of fading coefficients (i.e., $\sigma_{a}$ ) for indoor wireless channels fall in the range of 3-5 $\mathrm{dB}$ [14].

To reflect the effects of relays' location on the performance of cooperative schemes, we further incorporate the path loss effects in our transmission model. Assume that source, relay, and destination terminals are located in a two-dimensional plane where $d_{S D}, d_{S R}$, and $d_{R D}$ denote the distances of the underlying $\mathrm{S} \rightarrow \mathrm{D}, \mathrm{S} \rightarrow \mathrm{R}$, and $\mathrm{R} \rightarrow \mathrm{D}$ links respectively and $\theta$ is the angle between lines $\mathrm{S} \rightarrow \mathrm{R}$ and $\mathrm{R} \rightarrow \mathrm{D}$. The path loss of a link $\mathrm{A} \rightarrow \mathrm{B}$ is given by $P L(A, B)=K / d_{A B}^{\alpha}$ [18] where $K$ is a constant depending on the propagation environment, $d_{A B}$ denotes the Euclidean distance between nodes $\mathrm{A}$ and $\mathrm{B}$, and $\alpha$ is the path loss coefficient. In indoor wireless channels, $\alpha$ varies from 1.6 to 6 [18]. Normalizing the path loss by $P L(S, D)$ associated with the direct link, the relative geometrical gain of $\mathrm{S} \rightarrow \mathrm{R}$ and $\mathrm{R} \rightarrow \mathrm{D}$ links are defined, respectively, as [19] $G_{S R}=P L(S, R) / P L(S, D)=\left(d_{S D} / d_{S R}\right)^{\alpha}$ and $G_{R D}=P L(R, D) / P L(S, D)=\left(d_{S D} / d_{R D}\right)^{\alpha}$, which can be related to each other as $G_{R D}^{2 / \alpha}+G_{S R}^{2 / \alpha}-2 G_{R D}^{1 / \alpha} G_{S R}^{1 / \alpha} \cos \theta=$ $G_{R D}^{2 / \alpha} G_{S R}^{2 / \alpha}$. Furthermore, the path loss ratio can be defined as

$$
\beta=\frac{G_{S R}}{G_{R D}}=\left(\frac{d_{R D}}{d_{S R}}\right)^{\alpha}
$$

which indicates the location of the relay relative to the source and destination. The smaller the ratio is, the closer the relay is located to the destination. The particular case of $\beta=0$ $\mathrm{dB}$ means both source and destination terminals have the same distance to the relay. In the following, we introduce the received signal models for Protocols I, II, and III assuming amplify-and-forward relaying and the related power allocation parameters.

Protocol I: In Protocol I, the source terminal communicates with the relay and destination terminals during the first time slot, i.e., broadcasting phase. In the second time slot, i.e., relaying phase, both the relay and source terminals communicate with the destination terminal. In the corresponding power allocation problem, we need to identify three variables; namely, the power used by the source terminal in the broadcasting phase (i.e., $P_{S 1}$ ), the power used by the source terminal in the relaying phase (i.e., $P_{S 2}$ ), and the power used by the relay terminal $\left(P_{R 2}\right)$. The overall energy used by both source and relay terminals is $2 E$ during two time slots, therefore $P_{s 1}+P_{s 2}+P_{R 2}=2 E$. We introduce two optimization parameters, namely $K_{T}$ which represents the fraction of power dedicated to the broadcasting phase and $K_{S}$ which represents the fraction of power allocated to the source terminal in the relaying phase. It can be then easily verified that $P_{S 1}=2 K_{T} E, P_{S 2}=2\left(1-K_{T}\right) K_{S} E$, and $P_{R 2}=2\left(1-K_{T}\right)\left(1-K_{S}\right) E$ for $0 \leq K_{T}, K_{S} \leq 1$. For equal power allocation, $K_{T}$ and $K_{S}$ are simply set to 0.5 .

Let $x_{1}$ denote the transmitted signal with power $P_{S 1}=$ $2 K_{T} E$ in the first time slot. Assuming M-PSK (phase shift keying) modulation with normalized unit energy for the signals and considering path-loss effects, the received signals at the relay and destination are respectively given as

$$
r_{R}=\sqrt{2 G_{S R} K_{T} E} h_{S R} x_{1}+n_{R},
$$




$$
r_{D, 1}=\sqrt{2 K_{T} E} h_{S D} x_{1}+n_{D, 1}
$$

In the second time slot, the source terminal transmits $x_{2}$ using power $P_{S 2}=2\left(1-K_{T}\right) K_{S} E$. At the relay terminal, the received signal $r_{R}$ is normalized by a factor of $E\left[\left|r_{R}\right|^{2}\right]=$ $\sqrt{2 G_{S R} K_{T} E+N_{0}}$ to ensure the unity of average energy and retransmitted using $P_{R 2}=2\left(1-K_{T}\right)\left(1-K_{S}\right) E$ in the second time slot. Therefore, the received signal at the destination terminal is the superposition of transmitted signals by the relay and source terminals resulting in

$$
\begin{aligned}
r_{D, 2}^{\prime}= & \sqrt{2 G_{R D}\left(1-K_{T}\right)\left(1-K_{S}\right) E} h_{R D} \frac{r_{R}}{\sqrt{E\left[\left|r_{R}\right|^{2}\right]}} \\
& +\sqrt{2\left(1-K_{T}\right) K_{S} E} h_{S D} x_{2}+n_{D, 2}^{\prime} .
\end{aligned}
$$

In (4)-(6), $n_{R}, n_{D, 1}$, and $n_{D, 2}^{\prime}$ are independent complex Gaussian random variables with zero mean and variance $N_{0} / 2$ per dimension, which model additive noise terms. Replacing the normalizing term $E\left[\left|r_{R}\right|^{2}\right]=\sqrt{2 G_{S R} K_{T} E+N_{0}}$ in (6) we obtain

$$
\begin{aligned}
r_{D, 2}^{\prime}= & 2 E \sqrt{\frac{G_{S R} G_{R D}\left(1-K_{T}\right)\left(1-K_{S}\right) K_{S}}{2 G_{S R} K_{T} E+N_{0}}} h_{R D} h_{S R} x_{1} \\
& +\sqrt{2\left(1-K_{T}\right) K_{S} E} h_{S D} x_{2}+\tilde{n}
\end{aligned}
$$

where the effective noise term $\tilde{n}$ is defined as

$\tilde{n}=\sqrt{\frac{2 G_{R D}\left(1-K_{T}\right)\left(1-K_{S}\right) K_{T} E}{2 G_{S R} K_{T} E+N_{0}}} h_{R D} n_{R}+n_{D, 2}^{\prime}$.

Conditioned on $h_{R D}, \tilde{n}$ is a zero mean complex Gaussian random variable with variance of

$$
\begin{aligned}
\sigma_{\tilde{n} \mid h_{R D}}^{2} & =E\left[|\tilde{n}|^{2} \mid h_{R D}\right] \\
& =\left(1+\frac{2 G_{R D}\left(1-K_{T}\right)\left(1-K_{S}\right) E h_{R D}^{2}}{2 G_{S R} K_{T} E+N_{0}}\right) N_{0} .
\end{aligned}
$$

We assume that the destination terminal normalizes the received signal given by (7) with $\sqrt{1+2 G_{R D}\left(1-K_{T}\right)\left(1-K_{S}\right) E h_{R D}^{2} /\left(2 G_{S R} K_{T} E+N_{0}\right)}$, resulting in ${ }^{1}$

$$
r_{D, 2}=\sqrt{\gamma_{1}} \sqrt{E} h_{R D} h_{S R} x_{1}+\sqrt{\gamma_{2}} \sqrt{E} h_{S D} x_{2}+n_{D, 2}
$$

where $n_{D, 2}$ is complex Gaussian random variable with zero mean and variance $N_{0} / 2$ per dimension. In (10), $\gamma_{1}$ and $\gamma_{2}$ are defined as $\gamma_{1}=2 G_{S R} K_{T} /\left(h_{R D}{ }^{2}+A_{1}\right)$ and $\gamma_{2}=$ $A_{2} /\left(h_{R D}{ }^{2}+A_{1}\right)$, respectively, where

$$
A_{1}=\frac{\left(2 G_{S R} K_{T} S N R+1\right)}{\left[2 G_{R D}\left(1-K_{T}\right)\left(1-K_{S}\right) S N R\right]}
$$

$$
A_{2}=K_{S}\left(2 G_{S R} K_{T} S N R+1\right) /\left[G_{R D}\left(1-K_{S}\right) S N R\right]
$$

with $S N R=E / N_{0}$. After setting up the relay-assisted transmission model for Protocol I given by (5) and (10), we now introduce space-time coding across the transmitted signals $x_{1}$ and $x_{2}$. For the case of single relay deployment as considered here, we use STBC (space-time block code) designed for two transmit antennas, i.e., Alamouti's scheme.

\footnotetext{
${ }^{1}$ This does not change the signal-to-noise ratio, but simplifies the ensuing presentation [8].
}

The received signals at the destination terminal over four time slots can be written in a matrix form as

$$
\begin{aligned}
& \underbrace{\left[\begin{array}{l}
r_{D, 1} \\
r_{D, 2} \\
r_{D, 3} \\
r_{D, 4}
\end{array}\right]^{T}}_{\mathbf{r}}=\underbrace{\left[\begin{array}{ll}
h_{S D} & h_{S R} h_{R D}
\end{array}\right]}_{\mathbf{h}} \mathbf{X}+\underbrace{\left[\begin{array}{c}
n_{D, 1} \\
n_{D, 2} \\
n_{D, 3} \\
n_{D, 4}
\end{array}\right]}_{\mathbf{n}} \\
& \mathbf{X}^{T}=\left[\begin{array}{cc}
\sqrt{2 K_{T}} \sqrt{E} x_{1} & 0 \\
\sqrt{\gamma_{2}} \sqrt{E} x_{2} & \sqrt{\gamma_{1}} \sqrt{E} x_{1} \\
\sqrt{2 K_{T}} \sqrt{E} x_{2}^{*} & 0 \\
\sqrt{\gamma_{2}} \sqrt{E} x_{1}^{*} & -\sqrt{\gamma_{1}} \sqrt{E} x_{2}^{*}
\end{array}\right] .
\end{aligned}
$$

Protocol II: This protocol is a distributed SIMO implementation and does not involve transmit diversity. Therefore, unlike Protocol I which relies on two optimization parameters $K_{T}$ and $K_{S}$, only $K_{T}$ is relevant for Protocol II optimization. Considering path loss effects, the received signals at the relay and destination in the first time slot are given as

$$
\begin{gathered}
r_{R}=\sqrt{2 G_{S R} K_{T} E} h_{S R} x_{1}+n_{R}, \\
r_{D, 1}=\sqrt{2 K_{T} E} h_{S D} x_{1}+n_{D, 1} .
\end{gathered}
$$

For the second time slot, the received signal at the destination is given by

$$
r_{D, 2}^{\prime}=\sqrt{2 G_{R D}\left(1-K_{T}\right) E} h_{R D} \frac{r_{R}}{\sqrt{E\left[\left|r_{R}\right|^{2}\right]}}+n_{D, 2}^{\prime} .
$$

Replacing (14) and $E\left[\left|r_{R}\right|^{2}\right]=\sqrt{2 G_{S R} K_{T} E+N_{0}}$ in (16) and further normalizing the resulting signal such that the additive noise term has a variance of $N_{0}$ [8], we have

$$
r_{D, 2}=\sqrt{\gamma_{3}} \sqrt{E} h_{S D} h_{R D} x+n_{D, 2}
$$

where $\gamma_{3}=2 G_{S R} K_{T} /\left(h_{R D}{ }^{2}+A_{3}\right)$ with $A_{3}=$ $\left(2 G_{S R} K_{T} S N R+1\right) /\left[2 G_{R D}\left(1-K_{T}\right) S N R\right]$. (15) and (17) can be written in matrix form yielding

$$
\begin{aligned}
& \underbrace{\left[\begin{array}{l}
r_{D, 1} \\
r_{D, 2}
\end{array}\right]^{T}}_{\mathbf{r}}=\underbrace{\left[\begin{array}{cc}
h_{S D} & h_{S R} h_{R D}
\end{array}\right]}_{\mathbf{h}} \mathbf{X}+\underbrace{\left[\begin{array}{c}
n_{D, 1} \\
n_{D, 2}
\end{array}\right]^{T}}_{\mathbf{n}} \\
& \mathbf{X}=\left[\begin{array}{cc}
\sqrt{2 K_{T}} \sqrt{E} x_{1} & 0 \\
0 & \sqrt{\gamma_{3}} \sqrt{E} x_{1}
\end{array}\right] .
\end{aligned}
$$

Protocol III: This protocol is identical to Protocol I apart from the fact that the destination terminal chooses not to receive the direct source-to-destination transmission during the first time slot for reasons which are possibly imposed from the upper-layer networking protocols. For example, the destination terminal may be engaged in data transmission to another terminal during the first time slot [8]. Following similar steps as those for Protocol I, we can obtain the received signals for this protocol. In matrix form, we have

$$
\begin{aligned}
& \underbrace{\left[\begin{array}{c}
r_{D, 2} \\
r_{D, 4}
\end{array}\right]^{T}}_{\mathbf{r}}=\underbrace{\left[\begin{array}{cc}
h_{S D} & h_{S R} h_{R D}
\end{array}\right]}_{\mathbf{h}} \mathbf{X}+\underbrace{\left[\begin{array}{c}
n_{D, 2} \\
n_{D, 4}
\end{array}\right]^{T}}_{\mathbf{n}} \\
& \mathbf{X}=\left[\begin{array}{cc}
\sqrt{\gamma_{2}} \sqrt{E} x_{2} & \sqrt{\gamma_{2}} \sqrt{E} x_{1}^{*} \\
\sqrt{\gamma_{1}} \sqrt{E} x_{1} & -\sqrt{\gamma_{1}} \sqrt{E} x_{2}^{*}
\end{array}\right] .
\end{aligned}
$$

which can be simply obtained from (13). 


\section{DERIVATION OF UPPER BOUNDS ON PEP}

PEP is the building block for the derivation of union bounds to the error probability. It is widely used in the literature to predict the attainable diversity order where the closedform error probability expressions are unavailable. Defining the transmitted codeword vector and the erroneously-decoded codeword vector as $\mathbf{X}$ and $\hat{\mathbf{X}}$, respectively, the conditional $\mathrm{PEP}$ is given as [1]

$$
\begin{aligned}
P(\mathbf{X} \rightarrow \hat{\mathbf{X}} \mid \mathbf{h})= & Q\left(\sqrt{\frac{d^{2}(\mathbf{X}, \hat{\mathbf{X}})}{2 N_{0}}}\right) \\
& \leq \frac{1}{2} \exp \left(-\frac{d^{2}(\mathbf{X}, \hat{\mathbf{X}})}{4 N_{0}}\right)
\end{aligned}
$$

assuming maximum likelihood (ML) decoding with perfect knowledge of the channel state information (CSI) at the receiver side. In $(20), d^{2}(\mathbf{X}, \hat{\mathbf{X}})$ denotes the Euclidean distance between $\mathbf{X}$ and $\hat{\mathbf{X}}$ (conditional on $\mathbf{h}$ ) and is given by

$$
d^{2}(\mathbf{X}, \hat{\mathbf{X}})=\mathbf{h}(\mathbf{X}-\hat{\mathbf{X}})(\mathbf{X}-\hat{\mathbf{X}})^{\mathrm{H}} \mathbf{h}^{\mathrm{H}},
$$

where $\mathbf{X}$ is defined by (13), (18), and (19) for the cooperation protocols under consideration.

\section{A. PEP for Protocol I}

Replacing (13) in (21), the Euclidean distance for Protocol I is obtained as

$$
d^{2}(\mathbf{X}, \hat{\mathbf{X}})=\left(e^{\frac{2 a_{S D}}{\xi}}\left(2 K_{T}+\gamma_{2}\right)+e^{\frac{2\left(a_{S R}+a_{R D}\right)}{\xi}} \gamma_{1}\right) E \chi
$$

where $\chi=\left|\left(x_{1}-\hat{x}_{1}\right)\right|^{2}+\left|\left(x_{2}-\hat{x}_{2}\right)\right|^{2}$. Replacing (22) in (20) and averaging the resulting expression with respect to $a_{S D}$ and $a_{S R}$, we have

$$
\begin{aligned}
& P\left(\mathbf{X} \rightarrow \hat{\mathbf{X}} \mid a_{R D}\right) \leq \frac{1}{2} \frac{1}{\sqrt{2 \pi \sigma_{S D}^{2}}} \frac{1}{\sqrt{2 \pi \sigma_{S R}^{2}}} \\
& \times \int_{-\infty}^{+\infty} \exp \left(\frac{-E\left(2 K_{T}+\gamma_{2}\right) \chi}{4 N_{0}} e^{\frac{2 a_{S D}}{\xi}}\right) e^{\frac{-\left(a_{S D}+\sigma_{S D}^{2} / \xi\right)^{2}}{2 \sigma_{S D}^{2}}} d a_{S D} \\
& \times \int_{-\infty}^{+\infty} \exp \left(\frac{-E \gamma_{1} \chi}{4 N_{0}} e^{\frac{2\left(a_{S R}+a_{R D}\right)}{\xi}}\right) e^{-\frac{\left(a_{S R}+\sigma_{S R}^{2} / \xi\right)^{2}}{2 \sigma_{S R}^{2}}} d a_{S R} .
\end{aligned}
$$

Substituting $y=\exp \left(a_{S D} / \xi\right)$ and $z=\exp \left(a_{S R} / \xi\right)$ in (23), we obtain

$$
\begin{aligned}
& P\left(\mathbf{X} \rightarrow \hat{\mathbf{X}} \mid a_{R D}\right) \leq \frac{1}{2} \frac{1}{\sqrt{2 \pi \sigma_{S D}^{2} / \xi^{2}}} \frac{1}{\sqrt{2 \pi \sigma_{S R}^{2} / \xi^{2}}} \\
& \times \int_{0}^{+\infty} \frac{1}{y} \exp \left(-\frac{E\left(2 K_{T}+\gamma_{2}\right) \chi}{4 N_{0}} y^{2}\right) e^{-\frac{\left(\ln y+\sigma_{S D}^{2} / \xi^{2}\right)^{2}}{2 \sigma_{S D}^{2} / \xi^{2}}} d y \\
& \times \int_{0}^{+\infty} \frac{1}{z} \exp \left(-\frac{E \gamma_{1} \chi}{4 N_{0}} e^{\frac{2 a_{R D}}{\xi}} z^{2}\right) e^{-\frac{\left(\ln z+\sigma_{S R}^{2} / \xi^{2}\right)^{2}}{2 \sigma_{S R}^{2} / \xi^{2}}} d z \\
& =\frac{1}{2} \operatorname{Fr}\left(\frac{E\left(2 K_{T}+\gamma_{2}\right) \chi}{4 N_{0}} ; 0, \frac{\sigma_{S D}}{\xi}\right) \operatorname{Fr}\left(\frac{E \gamma_{1} \chi}{4 N_{0}} e^{\frac{2 a_{R D}}{\xi}} ; 0, \frac{\sigma_{S R}}{\xi}\right)
\end{aligned}
$$

where $\operatorname{Fr}(\alpha ; \beta, \sigma)$ is the frustration function defined as [20]

$$
\begin{aligned}
\operatorname{Fr}(\alpha ; \beta, \sigma)= & \int_{-\infty}^{\infty} \frac{1}{\sqrt{2 \pi \sigma^{2}}} I_{0}(2 \beta u \sqrt{\alpha}) \exp \left(-\alpha u^{2}\right) \\
& \times \exp \left(-\left(\ln u+\sigma^{2}\right)^{2} / 2 \sigma^{2}\right) d u
\end{aligned}
$$

and $I_{0}(\cdot)$ is the modified Bessel function of order zero. Further averaging (24) with respect to $a_{R D}$, we obtain unconditional
PEP expression as

$$
\begin{aligned}
& P(\mathbf{X} \rightarrow \hat{\mathbf{X}}) \leq \frac{1}{2} \int_{-\infty}^{+\infty} \frac{1}{\sqrt{2 \pi \sigma_{R D}^{2}}} \exp \left(-\frac{\left(a_{R D}+\sigma_{R D}^{2} / \xi\right)^{2}}{2 \sigma_{R D}^{2}}\right) \\
& \times \operatorname{Fr}\left(\frac{E\left(2 K_{T}+\gamma_{2}\right) \chi}{4 N_{0}} ; 0, \frac{\sigma_{S D}}{\xi}\right) \operatorname{Fr}\left(\frac{E \gamma_{1} \chi}{4 N_{0}} e^{\frac{2 a_{R D}}{\xi}} ; 0, \frac{\sigma_{S R}}{\xi}\right) d a_{R D}
\end{aligned}
$$

Let $u=\left(a_{R D}+\sigma_{R D}^{2} / \xi\right) / \sqrt{2 \sigma_{R D}^{2}}$. After substituting the variable $a_{R D}=u \sqrt{2 \sigma_{R D}^{2}}-\sigma_{R D}^{2} / \xi$ in (26), we have

$$
\begin{aligned}
P(\mathbf{X} \rightarrow \hat{\mathbf{X}}) \leq \frac{1}{2 \sqrt{\pi}} & \int_{-\infty}^{\infty} \exp \left(-u^{2}\right) \operatorname{Fr}\left(\frac{E B_{1} \chi}{4 N_{0}} ; 0, \frac{\sigma_{S R}}{\xi}\right) \\
& \times \operatorname{Fr}\left(\frac{E\left(2 K_{T}+B_{2}\right) \chi}{4 N_{0}} ; 0, \frac{\sigma_{S D}}{\xi}\right) d u
\end{aligned}
$$

where $B_{1}$ and $B_{2}$ are defined as

$$
B_{1}=\frac{2 G_{S R} K_{T}}{1+\frac{A_{1}}{\exp \left[u \sqrt{8 \sigma_{R D}^{2} / \xi^{2}}-2 \sigma_{R D}^{2} / \xi^{2}\right]}}
$$

$$
B_{2}=\frac{A_{2}}{A_{1}+\exp \left[u \sqrt{8 \sigma_{R D}^{2} / \xi^{2}}-2 \sigma_{R D}^{2} / \xi^{2}\right]} .
$$

A closed-form solution for (27) is unfortunately not available. However, imposing some assumptions on $\beta$ which reflects the relay location, we are able to simplify this expression and obtain an expression in terms of frustration function. In particular, we consider two cases where either the relay is located very close to source, i.e., $\beta>>1$, or very close to destination, i.e., $\beta<<1$. Under the assumption of $\beta>>1$, geometrical gains are $G_{S R}>>1$ and $G_{R D} \approx 1$, hence $\gamma_{1}$ and $\gamma_{2}$ can be approximated as $\gamma_{1} \cong 2\left(1-K_{T}\right)\left(1-K_{S}\right)$ and $\gamma_{2} \cong 2 K_{S}\left(1-K_{T}\right)$. Replacing (22) in (20), and averaging the resulting expression with respect to $a_{S D}$ and $a_{S}=a_{S R}+$ $a_{R D}$ which are normally distributed with standard deviations $\sigma_{S D}$ and $\sqrt{\sigma_{S R}^{2}+\sigma_{R D}^{2}}$, we have

$$
\begin{aligned}
P(\mathbf{X} \rightarrow & \hat{\mathbf{X}}) \leq \frac{1}{2} \operatorname{Fr}\left(\frac{\left[K_{T}+K_{S}\left(1-K_{T}\right)\right] E \chi}{2 N_{0}} ; 0, \frac{\sigma_{S D}}{\xi}\right) \\
& \times \operatorname{Fr}\left(\frac{\left(1-K_{T}\right)\left(1-K_{S}\right) E \chi}{2 N_{0}} ; 0, \frac{\sqrt{\sigma_{S R}^{2}+\sigma_{R D}^{2}}}{\xi}\right) .
\end{aligned}
$$

On the other hand, under the assumption of $\beta<<1, G_{R D}$ is much larger than $G_{S R} \cong 1$, hence $\gamma_{1}$ and $\gamma_{2}$ can be approximated as $\gamma_{1} \cong 2 G_{S R} K_{T} e^{-2 a_{R D}}$ and $\gamma_{2} \cong 0$. For this case, we obtain

$$
\begin{aligned}
P(\mathbf{X} \rightarrow \hat{\mathbf{X}}) \leq & \frac{1}{2} \operatorname{Fr}\left(\frac{K_{T} E \chi}{2 N_{0}} ; 0, \frac{\sigma_{S D}}{\xi}\right) \\
& \times \operatorname{Fr}\left(\frac{K_{T} E \chi}{2 N_{0}} ; 0, \frac{\sigma_{S R}}{\xi}\right)
\end{aligned}
$$

\section{B. PEP for Protocol II}

Replacing (18) in (21), the Euclidean distance for Protocol II is given by

$$
d^{2}(\mathbf{X}, \hat{\mathbf{X}})=e^{2 a_{S D} / \xi} 2 K_{T} E \chi+e^{2 a_{S R} / \xi} e^{2 a_{R D} / \xi} E \gamma_{1} \chi
$$

where $\chi=\left|x_{1}-\hat{x}_{1}\right|^{2}$. Replacing (32) in (20) and averaging the resulting expression with respect to $a_{S D}, a_{R D}$, and $a_{S R}$, we obtain the PEP expression as

$$
\begin{aligned}
P(\mathbf{X} \rightarrow \hat{\mathbf{X}}) \leq & \frac{1}{2 \sqrt{\pi}} \operatorname{Fr}\left(\frac{K_{T} E \chi}{2 N_{0}} ; 0, \frac{\sigma_{S D}}{\xi}\right) \\
& \times \int_{-\infty}^{\infty} \exp \left(-u^{2}\right) \operatorname{Fr}\left(\frac{E B_{1} \chi}{4 N_{0}} ; 0, \frac{\sigma_{S R}}{\xi}\right) d u
\end{aligned}
$$


where $B_{1}$ has been previously defined by (28). Similar to (27), a closed-form solution for (33) is not available. However, for $\beta>>1$ and $\beta<<1$, we can obtain simplified expressions such as

$$
\begin{aligned}
P(\mathbf{X} \rightarrow \hat{\mathbf{X}}) \leq & \frac{1}{2} \operatorname{Fr}\left(\frac{K_{T} E \chi}{2 N_{0}} ; 0, \frac{\sigma_{S D}}{\xi}\right) \\
\times & \operatorname{Fr}\left(\frac{\left(1-K_{T}\right) E \chi}{2 N_{0}} ; 0, \frac{\sigma_{S R}^{2}+\sigma_{R D}^{2}}{\xi}\right) \\
P(\mathbf{X} \rightarrow \hat{\mathbf{X}}) \leq & \frac{1}{2} \operatorname{Fr}\left(\frac{K_{T} E \chi}{2 N_{0}} ; 0, \frac{\sigma_{S D}}{\xi}\right) \\
& \times \operatorname{Fr}\left(\frac{K_{T} E \chi}{2 N_{0}} ; 0, \frac{\sigma_{S R}}{\xi}\right) .
\end{aligned}
$$

\section{PEP for Protocol III}

Replacing (19) in (21), the Euclidean distance for Protocol III is given by

$$
d^{2}(\mathbf{X}, \hat{\mathbf{X}})=e^{2 a_{S D} / \xi} E \gamma_{2} \chi+e^{2 a_{S R} / \xi} e^{2 a_{R D} / \xi} E \gamma_{1} \chi
$$

Replacing (36) in (20) and averaging the resulting expression with respect to $a_{S D}, a_{S R}$, and $a_{R D}$, the PEP expression is upper-bounded as

$$
\begin{aligned}
P(\mathbf{X} \rightarrow \hat{\mathbf{X}}) \leq \frac{1}{2 \sqrt{\pi}} & \int_{-\infty}^{\infty} \exp \left(-u^{2}\right) \operatorname{Fr}\left(\frac{E B_{2} \chi}{4 N_{0}} ; 0, \frac{\sigma_{S D}}{\xi}\right) \\
& \times \operatorname{Fr}\left(\frac{E B_{1} \chi}{4 N_{0}} ; 0, \frac{\sigma_{S R}}{\xi}\right) d u
\end{aligned}
$$

where $B_{1}$ and $B_{2}$ have been earlier defined by (28) and (29). For $\beta>>1$ and $\beta<<1$, we obtain

$$
\begin{aligned}
& P(\mathbf{X} \rightarrow \hat{\mathbf{X}}) \leq \frac{1}{2} \operatorname{Fr}\left(\frac{K_{S}\left(1-K_{T}\right) E \chi}{2 N_{0}} ; 0, \frac{\sigma_{S D}}{\xi}\right) \\
& \times \operatorname{Fr}\left(\frac{\left(1-K_{S}\right)\left(1-K_{T}\right) E \chi}{2 N_{0}} ; 0, \frac{\sigma_{S R}^{2}+\sigma_{R D}^{2}}{\xi}\right), \\
& P(\mathbf{X} \rightarrow \hat{\mathbf{X}}) \leq \frac{1}{2} \operatorname{Fr}\left(\frac{K_{T} E \chi}{2 N_{0}} ; 0, \frac{\sigma_{S R}}{\xi}\right) .
\end{aligned}
$$

\section{Performance Optimization through Power ALLOCATION}

It has been demonstrated in recent papers [21]-[26] that the performance of cooperative communication schemes can be significantly improved by properly allocating the overall power among cooperating nodes rather than the equal power allocation. In this section, we investigate the optimal power allocation for log-normal fading relay channels aiming to minimize the BER performance. A union bound on the BER performance is given by [27]

$$
P_{b} \leq \frac{1}{n} \sum_{\mathbf{X}} p(\mathbf{X}) \sum_{\mathbf{X} \neq \hat{\mathbf{X}}} q(\mathbf{X} \rightarrow \hat{\mathbf{X}}) P(\mathbf{X} \rightarrow \hat{\mathbf{X}})
$$

where $p(\mathbf{X})$ is the probability that codeword $\mathbf{X}$ is transmitted, $q(\mathbf{X} \rightarrow \hat{\mathbf{X}})$ is the number of information bit errors in choosing another codeword $\hat{\mathbf{X}}$ instead of the original one, $n$ is the number of information bits per transmission, and $P(\mathbf{X} \rightarrow \hat{\mathbf{X}})$ is the PEP. Replacing the derived PEP expressions in (40), the objective functions for the protocols under consideration are obtained. The specific form of BER expressions depends on the modulation scheme and underlying code. For example, if BPSK is used as the modulation scheme, upper bounds on
BER for distributed Alamouti's scheme can be calculated for Protocols I, II, and III, respectively, as

$$
\begin{gathered}
\Gamma_{\mathrm{I}}\left(K_{T}, K_{S}\right)=\Psi_{\mathrm{I}}(2)+\Psi_{\mathrm{I}}(4), \\
\Gamma_{\mathrm{II}}\left(K_{T}, K_{S}\right)=\Psi_{\mathrm{II}}(4), \\
\Gamma_{\mathrm{III}}\left(K_{T}, K_{S}\right)=\Psi_{\mathrm{III}}(2)+\Psi_{\mathrm{III}}(4),
\end{gathered}
$$

where $\Psi_{i}(\chi)$ is the PEP expression for $i^{\text {th }}$ cooperative protocol which are given by the (27), (33), and (37). $\Psi_{i}(\chi)$ is obviously dependent on $K_{T}$ and $K_{S}$. If QPSK is used, the upper bounds on BER are given as

$$
\begin{gathered}
\Gamma_{\mathrm{I}}\left(K_{T}, K_{S}\right)=\Psi_{\mathrm{I}}(2)+3 \Psi_{\mathrm{I}}(4)+3 \Psi_{\mathrm{I}}(6)+\Psi_{\mathrm{I}}(8), \\
\Gamma_{\mathrm{II}}\left(K_{T}, K_{S}\right)=\Psi_{\mathrm{II}}(2)+\Psi_{\mathrm{II}}(4), \\
\Gamma_{\mathrm{III}}\left(K_{T}, K_{S}\right)=\Psi_{\mathrm{III}}(2)+3 \Psi_{\mathrm{III}}(4)+3 \Psi_{\mathrm{III}}(6)+\Psi_{\mathrm{III}}(8) .
\end{gathered}
$$

Similar bounds can be found in a straightforward manner for higher-order PSK schemes. We need to minimize the resulting objective functions $\Gamma_{i}$ with respect to the power allocation parameters $K_{T}$ and $K_{S}$ subject to the constraints $0 \leq K_{T}, K_{S} \leq 1$. Hence the optimization problem can be expressed as

$$
\begin{array}{ll}
\text { Minimize } & \Gamma_{\mathrm{i}}\left(K_{T}, K_{S}\right) \\
\text { subject to } & 0 \leq K_{T}, K_{S} \leq 1 .
\end{array}
$$

Since no closed-form solution is available, we resort to numerical methods to solve this optimization problem. In particular, we use "fmincon" command available in MATLAB optimization toolbox [28]. This command is designed to find the minimum of constrained nonlinear multivariable function. It implements a sequential quadratic programming (SQP) algorithm. At each iteration of the algorithm, an approximation of the Hessian of the Lagrangian function is calculated using a quasi-Newton updating method. This is then used to generate a quadratic programming sub-problem whose solution is used to form a search direction for a line search procedure. We also note that the solution of this optimization problem is a global minimum because the objective function is convex with respect to the optimization variables of $K_{T}$ and $K_{S}$. This can be readily verified for scenarios with $\beta<<1$ (see Appendix I). For the other relay locations, the problem becomes more complicated and it is very difficult, if not impossible, to present an analytical verification for convexity of the objective functions under consideration. We have, however, numerically verified the convexity in these cases for all considered SNR values. In Table I, we present optimized values of $K_{T}$ and $K_{S}$ for Protocols I, II, III assuming QPSK, $\theta=\pi, \sigma_{a}=4$, and path loss ratios of $\beta=-40 \mathrm{~dB}, 0 \mathrm{~dB}, 40 \mathrm{~dB}$. For $\beta=-40 \mathrm{~dB}$ (i.e., relay is very close to the destination), we observe that $K_{T}$ and $K_{S}$ converge to 1 and 0 , respectively. Convergence of $K_{T}$ to one can be also easily verified through PEP expressions of (31), (35), (39) noting that frustration function is a decreasing continuous function with respect to its first argument. These particular optimum values of $K_{T}$ and $K_{S}$ indicate that it is better to spend most of the overall power in broadcasting phase and rest of the power should be dedicated to the relay terminal 
TABLE I

OPTIMUM POWER ALLOCATION PARAMETERS FOR PROTOCOLS I, II, III.

(a) Protocol I

\begin{tabular}{|c||c|c|c|c|c|c|}
\hline \multicolumn{1}{|c||}{} & \multicolumn{7}{c|}{$G_{S R} / G_{R D}$} \\
\cline { 2 - 7 }$[\mathrm{dB}]$ & $-40 \mathrm{~dB}$ & \multicolumn{2}{c|}{$0 \mathrm{~dB}$} & \multicolumn{2}{c|}{$40 \mathrm{~dB}$} \\
\cline { 2 - 7 } & $K_{T}$ & $K_{S}$ & $K_{T}$ & $K_{S}$ & $K_{T}$ & $K_{S}$ \\
\hline 0 & 0.9872 & 0 & 0.6851 & 0 & 0.4639 & 0.4643 \\
5 & 0.9886 & 0 & 0.6656 & 0 & 0.4955 & 0.4175 \\
10 & 0.9890 & 0 & 0.6534 & 0 & 0.4540 & 0.4539 \\
15 & 0.9884 & 0 & 0.6590 & 0 & 0.4436 & 0.4428 \\
20 & 0.9890 & 0 & 0.6572 & 0 & 0.4204 & 0.4204 \\
25 & 0.9880 & 0 & 0.6560 & 0 & 0.4998 & 0.4998 \\
\hline
\end{tabular}

(b) Protocol II

\begin{tabular}{|c||c|c|c|}
\hline \multicolumn{1}{|c||}{\multirow{2}{*}{ SNR }} & \multicolumn{3}{c|}{$G_{S R} / G_{R D}$} \\
\cline { 2 - 4 }$[\mathrm{dB}]$ & $-40 \mathrm{~dB}$ & $0 \mathrm{~dB}$ & $40 \mathrm{~dB}$ \\
\cline { 2 - 4 } & $K_{T}$ & $K_{T}$ & $K_{T}$ \\
\hline 0 & 0.9873 & 0.6873 & 0.7171 \\
5 & 0.9887 & 0.6682 & 0.7087 \\
10 & 0.9891 & 0.6550 & 0.7010 \\
15 & 0.9891 & 0.6439 & 0.6945 \\
20 & 0.9890 & 0.6284 & 0.6879 \\
25 & 0.9890 & 0.5130 & 0.5280 \\
\hline
\end{tabular}

(c) Protocol III

\begin{tabular}{|c||c|c|c|c|c|c|}
\hline \multicolumn{1}{|c||}{\multirow{2}{*}{ SNR }} & \multicolumn{9}{c|}{$G_{S R} / G_{R D}$} \\
\cline { 2 - 7 }$[\mathrm{dB}]$ & \multicolumn{2}{|c|}{$-40 \mathrm{~dB}$} & \multicolumn{2}{c|}{$0 \mathrm{~dB}$} & \multicolumn{2}{c|}{$40 \mathrm{~dB}$} \\
\cline { 2 - 7 } & $K_{T}$ & $K_{S}$ & $K_{T}$ & $K_{S}$ & $K_{T}$ & $K_{S}$ \\
\hline 0 & 0.9818 & 0 & 0 & 1 & 0 & 1 \\
5 & 0.9838 & 0 & 0 & 1 & 0.0051 & 0.7070 \\
10 & 0.9845 & 0 & 0.2672 & 0.7700 & 0.0041 & 0.7012 \\
15 & 0.9846 & 0 & 0.2509 & 0.7660 & 0.0036 & 0.6779 \\
20 & 0.9840 & 0 & 0.2966 & 0.5620 & 0.0019 & 0.6801 \\
25 & 0.9767 & 0.9280 & 0.2280 & 0.6603 & 0.0020 & 0.6899 \\
\hline
\end{tabular}

in relaying phase. In effect, optimized version of Protocol I reduces to Protocol II and optimized version of Protocol III reduces to a non-cooperative double-hop transmission system. Under the assumption of $\beta=0 \mathrm{~dB}$ (i.e., the relay is located at the midpoint of the line connecting the source and destination terminals), the optimal values of $K_{S}$ for Protocol I are still equal to 0 while $K_{T}$ values indicate that roughly about 2/3 of the power should be spent in the broadcasting phase for Protocols I and II. In Protocol III, optimal $K_{T}$ tends to 0 and 1/4 for low and high SNR values, respectively. Under the assumption of $\beta=40 \mathrm{~dB}$ (i.e., relay is very close to the source), both $K_{T}$ and $K_{S}$ tend to 0.5 for Protocol I indicating equal power allocation is nearly optimal. For Protocol II, $K_{T}$ reduces from about $2 / 3$ to $1 / 2$ in the higher SNR region. For Protocol III, $K_{T}$ and $K_{S}$ tend to about $2 / 3$ and 0 , respectively.

To have insights into performance improvements through power allocation, we have calculated power efficiency gains achieved by optimum power allocation (OPA) for a target BER of $10^{-3}$ considering the range of $\beta=[-60 \mathrm{~dB}, 60 \mathrm{~dB}]$ and illustrated them in Fig. 1 along with equal power allocation (EPA). For Protocol I, Fig. 1.a indicates performance gains of $2.93 \mathrm{~dB}, 0.20 \mathrm{~dB}$ and $0.07 \mathrm{~dB}$ for $\beta=-40 \mathrm{~dB}, \beta=0 \mathrm{~dB}$, and $\beta=40 \mathrm{~dB}$, respectively. For Protocol II, similar performance improvements are observed up to $3 \mathrm{~dB}$ in the negative region of $\beta$. It is also observed that for both Protocols I and II, OPA and EPA performance curves converge in the region of positive $\beta$ values. This indicates that OPA is more rewarding for these two protocols in scenarios where relay is close to destination. The characteristics are rather different for Protocol III which benefits from OPA for both positive and negative $\beta$ values. Particularly, Fig. 1.c indicates performance gains of $2.85 \mathrm{~dB}$,

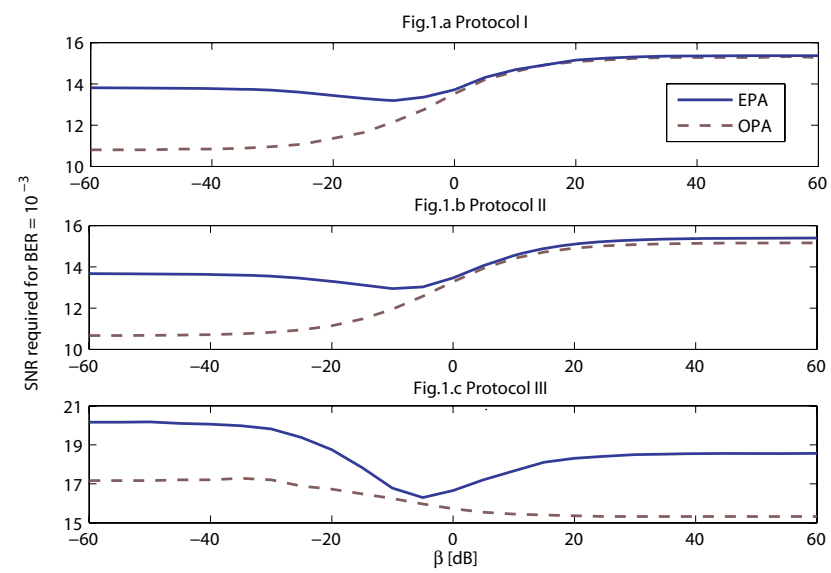

Fig. 1. Power efficiency gains for a target BER of $10^{-3}$ over log-normal fading channels.

$0.93 \mathrm{~dB}$, and $3.23 \mathrm{~dB}$ for $\beta=-40 \mathrm{~dB}, \beta=0 \mathrm{~dB}$, and $\beta=$ $40 \mathrm{~dB}$, respectively.

\section{Diversity GAin ANALYSis FOR LOG-NORMAL CHANNELS}

Diversity order is conventionally defined as the negative asymptotic slope of error probability versus $S N R=E / N_{0}$ on a log-log scale which can be expressed as

$$
d=-\lim _{S N R \rightarrow \infty} \frac{\log P_{b}}{\log S N R} .
$$

The diversity order for finite $S N R$ values can be further defined as [29]

$$
d_{f}(S N R)=-\frac{\partial \log P_{b}}{\partial \log S N R} .
$$

In log-normal fading channels, the decreasing rate of error probability does not converge to a finite value (see Appendix II) realizing effectively infinite diversity order based on the conventional definition in (48). Since conventional definition will be of no use for the channel model under consideration, we introduce the so-called relative diversity order $(R D O)$ as

$$
R D O(S N R)=\frac{d_{f}(S N R)}{d_{f, B M}(S N R)}=\frac{\frac{\partial \log P_{b}}{\partial \log S N R}}{\frac{\partial \log P_{b, B M}}{\partial \log S N R}}
$$

where we normalize the diversity gain defined as in (49) with respect to a benchmark scheme (i.e., $\left.d_{f, B M}\right)$. We further define the asymptotical relative diversity order $(A R D O)^{2}$ as

$$
A R D O=\lim _{S N R \rightarrow \infty} R D O(S N R) .
$$

Assuming direct transmission as benchmark scheme and BPSK as modulation scheme, a bound on the BER of the benchmark scheme can be found as

$$
\begin{aligned}
P_{b, \text { Direct }} & \leq \int_{-\infty}^{\infty} \frac{1}{2} e^{-h^{2} S N R} \frac{\xi}{\sqrt{2 \pi} \sigma_{S D} h} e^{-\frac{\left(20 \log _{10} h-m_{S D}\right)^{2}}{2 \sigma_{S D}^{2}}} d h \\
& =\frac{1}{2} \operatorname{Fr}\left(S N R ; 0, \frac{\sigma_{S D}}{\xi}\right) .
\end{aligned}
$$

\footnotetext{
${ }^{2}$ Considering direct transmission as the benchmark scheme, it can be readily shown that $A R D O$ and conventional diversity order coincide for Rayleigh fading channels.
} 


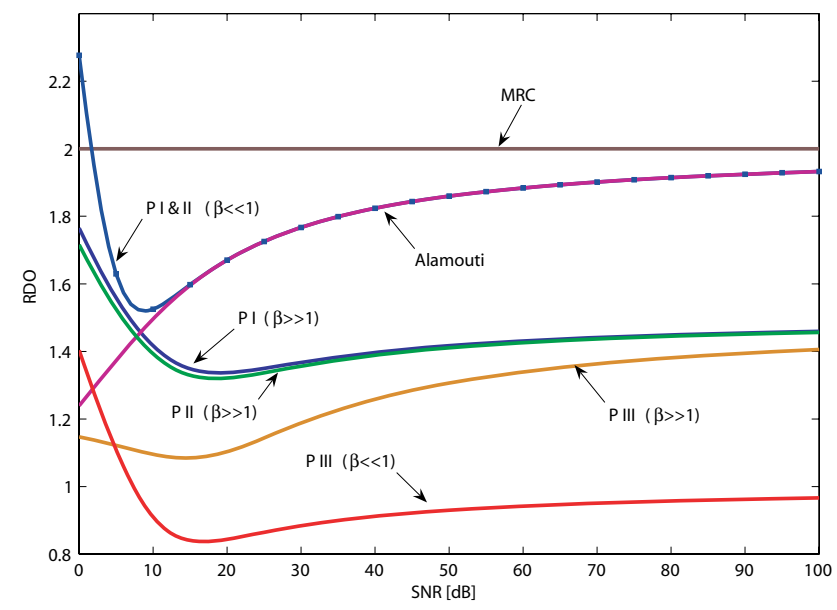

Fig. 2. Relative diversity order (RDO) of cooperative protocols with equal power allocation.

For maximal ratio combining (MRC) with two receive antennas and Alamouti scheme with two transmit antennas, BER can be upper bounded respectively as

$$
\begin{gathered}
P_{b, \mathrm{MRC}} \leq\left[\operatorname{Fr}\left(S N R ; 0, \frac{\sigma_{S D}}{\xi}\right)\right]^{2}, \\
P_{b, \text { Alamouti }} \leq\left[\operatorname{Fr}\left(S N R ; 0, \frac{\sigma_{S D}}{\xi}\right)\right]^{2} .
\end{gathered}
$$

It can be observed from (53) and (54) that there is a $3 \mathrm{~dB}$ difference in performance between Alamouti scheme and MRC over log-normal fading channels. This is similar to observations on the performance of these schemes over Rayleigh fading channels. These two schemes also provide the same $A R D O$ over log-normal fading channels. Replacing (52) and (53) into (51), it can be readily found that MRC yields $A R D O$ of 2 over log-normal fading channels. ARDO of Alamouti scheme can be obtained numerically and is also equal to 2 .

Figs. 2 and 3 illustrate the RDOs of Protocols I, II and III for $\beta<<1$ and $\beta>>1$ with EPA and OPA, respectively. Conventional MRC and Alamouti schemes with co-located antennas are included for comparison purposes. To make a fair comparison, we consider BPSK and QPSK modulations, respectively, for cooperative and conventional schemes which provide rates of $1 / 2$ and 1 . From Fig. 2, it is observed that Protocols I and II with EPA behave very similar to conventional Alamouti scheme and both converge to an $A R D O$ of 2 at very high SNR for $\beta<<1$ (i.e., relay is close to destination). The observation on similar performance can be further confirmed through PEP expressions; (31) demonstrates that the PEP expression for Protocol I becomes independent of $K_{S}$ for this particular relay location and becomes identical to (35) given for Protocol II.

From Fig. 3, it is observed that Protocols I and II with OPA are able to achieve an $A R D O$ of 2 similar to MRC. Note that the optimum value of $K_{T}$ is 1 for $\beta<<1$ which effectively reduces the PEP expressions derived for Protocols I and II (c.f., (31) and (35)) into a similar form of (53) derived for MRC scheme.

From Figs. 2 and 3, it is further observed that Protocol III for $\beta<<1$ achieves an ARDO of 1 (which can be

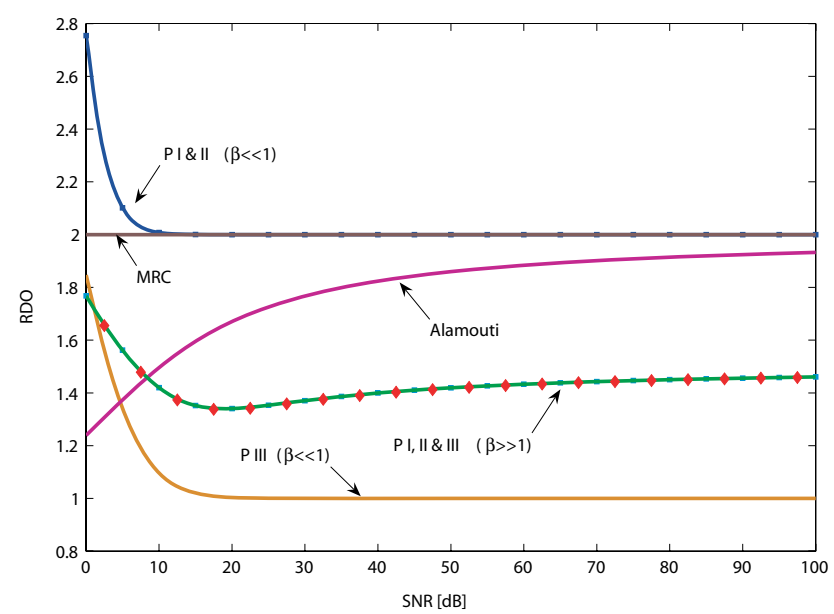

Fig. 3. Relative diversity order (RDO) of cooperative protocols with optimum power allocation.

readily verified from related PEP expression) under both EPA and OPA assumptions and is outperformed by the two other protocols for both cases. For $\beta>>1$, Protocols I, II, and III achieve the same $A R D O(\sim 1.5)$ under both EPA and OPA rules. Although it is observed that all three protocols have the same $R D O$ for the whole $S N R$ range under OPA, Protocols I and II outperform Protocol III in lower $S N R$ values under EPA.

\section{Simulation Results}

To further confirm the performance gains of OPA promised by the PEP expressions and associated $A R D O$ values, we have conducted a Monte Carlo simulation study to compare the BER performance of the considered protocols with EPA and OPA. We consider QPSK, $\theta=\pi, \sigma_{a}=4$, and path loss ratios of $\beta=-40 \mathrm{~dB},-10 \mathrm{~dB}, 0 \mathrm{~dB}, 10 \mathrm{~dB}, 40 \mathrm{~dB}$ in our simulations.

In Fig. 4, we consider Protocol I where we observe performance improvements of $2.81 \mathrm{~dB}, 1.13 \mathrm{~dB}, 0.33 \mathrm{~dB}$, $0.07 \mathrm{~dB}$ and $0.04 \mathrm{~dB}$ at a target BER of $10^{-3}$ for $\beta=$ $-40 \mathrm{~dB},-10 \mathrm{~dB}, 0 \mathrm{~dB}, 10 \mathrm{~dB}, 40 \mathrm{~dB}$ respectively. These results are very similar to performance gains predicted by the PEP expression of Protocol I (c.f., Fig. 1). Similar confirmation holds for the other two protocols and those simulation results are not included here due to space limitations.

Figs. 5-7 present performance comparisons of Protocols I, II, and III under EPA and OPA for $\beta=-40 \mathrm{~dB}, \beta=0 \mathrm{~dB}$, and $\beta=40 \mathrm{~dB}$, respectively. The performances of direct transmission (i.e., no relaying), Alamouti code, and MRC with two receive co-located antennas are further included to demonstrate how close the virtual antenna implementations can perform to their co-located counterparts.

In Fig. 5 illustrated for $\beta=-40 \mathrm{~dB}$, we observe that Protocols I and II provide nearly identical performance (very close to the performance of Alamouti scheme) under EPA and outperform Protocol III which performs even worse than the direct transmission scheme. Recall from Fig. 2 that $R D O$ of Protocol III is less than 1 for indicating its inferiority to the direct transmission for this particular relay location. This inefficiency of Protocol III results from lack of sourceto-destination transmission link in the first time slot; an 


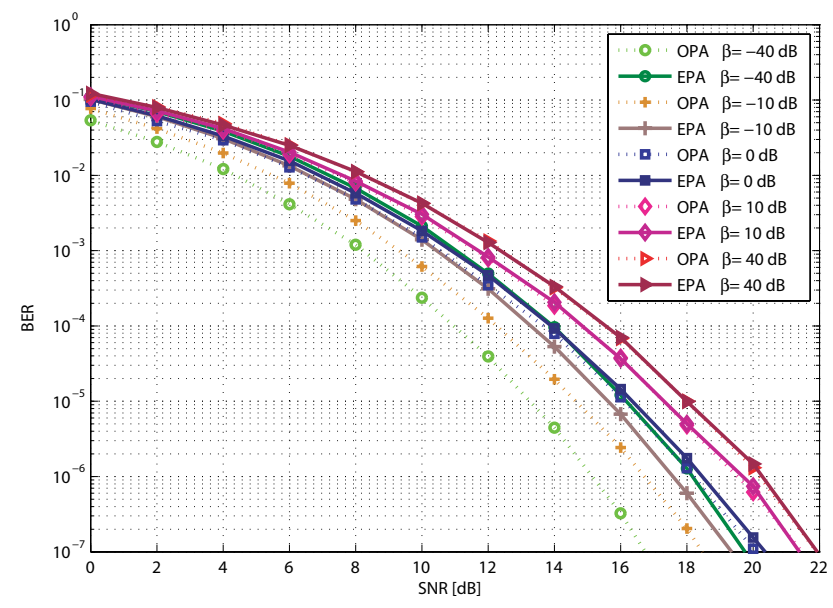

Fig. 4. Simulated BER performance of Protocol I for different values of $\beta$.

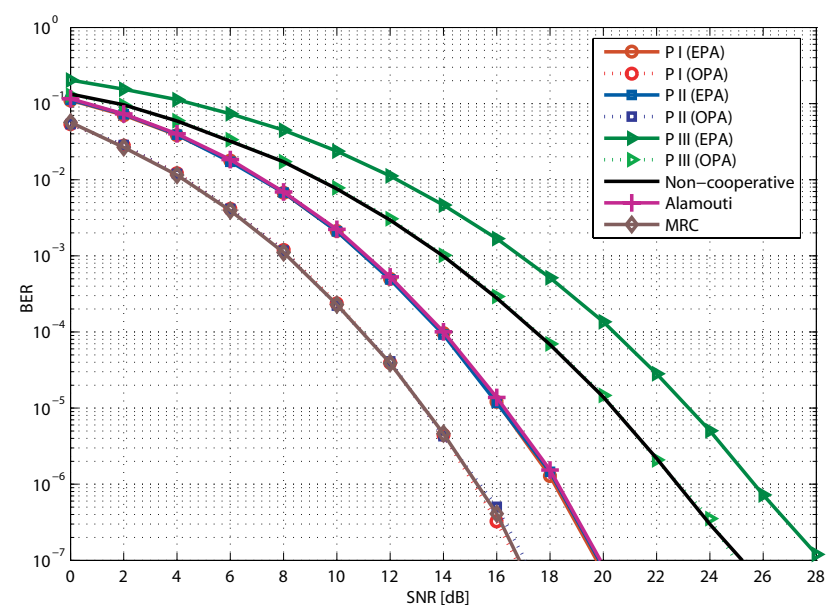

Fig. 5. Performance comparison of Protocols I, II, and III for $\beta=-40 \mathrm{~dB}$.

inherent disadvantage of Protocol III [8]. OPA improves the performance of the three protocols by $\sim 3 \mathrm{~dB}$. Protocols I and II with OPA present the same performance as MRC scheme and Protocol III is only able to achieve the performance of direct transmission. These observations confirm our earlier results on $R D O$ presented in Fig. 3 where we see identical $R D O$ curves for Protocols I and II converging to that of MRC scheme while $R D O$ curve for Protocol III converges to that of direct transmission.

From Fig. 6, we observe that the performance improvement of Protocols I and II through OPA is negligible for $\beta=40 \mathrm{~dB}$ (c.f., Figs. 1.a and 1.b). On the other hand, the performance of Protocol III is improved significantly and it operates at 1.3 $\mathrm{dB}$ away from the benchmark Alamouti scheme at $\mathrm{BER}=10^{-3}$ yielding identical performance with Protocols I and II for this particular relay location. This can be also confirmed from Fig. 3 where all corresponding $R D O$ curves coincide.

Fig. 7 illustrates that Protocols I and II perform nearly identical and are close to the performance of Alamouti scheme for $\beta=0 \mathrm{~dB}$ under both EPA and OPA. Similar to the case of $\beta=40 \mathrm{~dB}$, the performance improvement under OPA is not significant for these two protocols. Protocol III under EPA outperforms direct transmission for higher SNR values $(\mathrm{SNR}>13 \mathrm{~dB})$. OPA further improves the performance of Protocol III about $1 \mathrm{~dB}$ at $\mathrm{BER}=10^{-3}$.

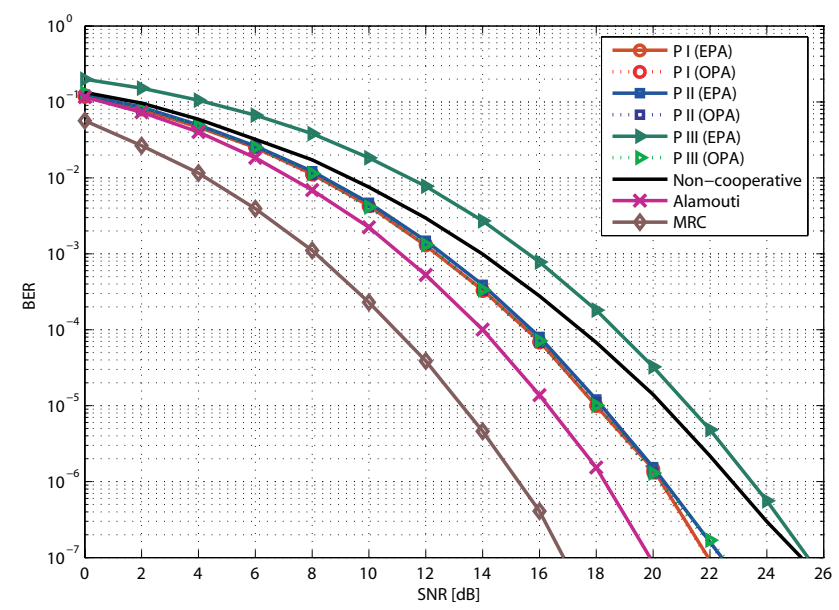

Fig. 6. Performance comparison of Protocols I, II, and III for $\beta=40 \mathrm{~dB}$.

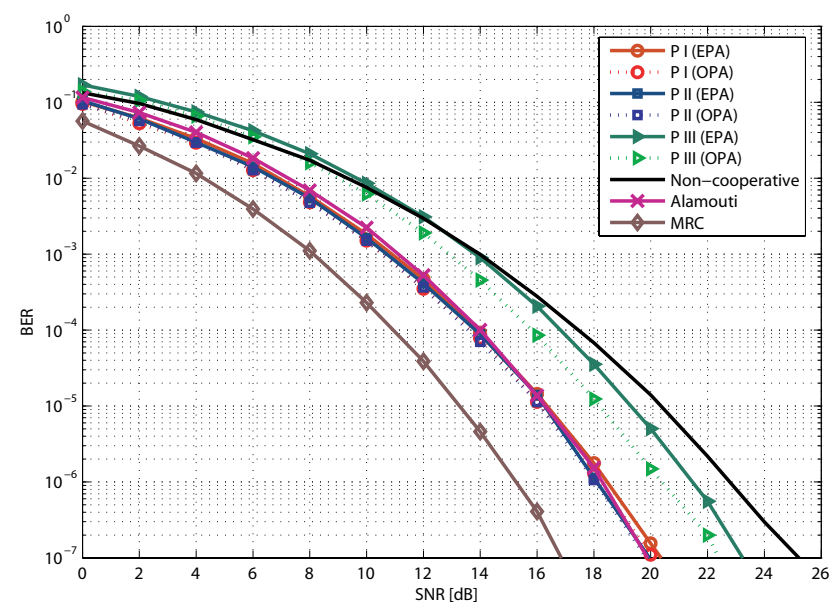

Fig. 7. Performance comparison of Protocols I, II, and III for $\beta=0 \mathrm{~dB}$.

\section{CONCLUSIONS}

In this paper, we have investigated the performance of cooperative diversity schemes over log-normal fading channels. Under Nabar et al.'s framework for single-relay networks [8], we have considered Protocols I, II, and III and derived upper bounds on PEP expressions for each of these protocols with amplify-and-forward relaying. We have further defined socalled relative diversity order and quantified diversity advantages for log-normal channels where conventional definition of diversity order can not be used. Based on a union bound on BER, we have further proposed OPA rules for performance improvement. Performance gains up to $3 \mathrm{~dB}$ at a target BER of $10^{-3}$ have been observed depending on the relay geometry and deployed protocol. It has been observed that optimized versions of Protocols I and II with single relay are able to provide similar performance to MRC with two co-located antennas for certain relay locations.

\section{APPENDIX I}

\section{Convexity of the OBJective Functions for $\beta<<1$}

The upper bound on PEP for Protocol III under the assumption of $\beta<<1$ is given by (39). This expression is in the form of a single frustration function and is a function of only the optimization parameter $K_{T}$. Substituting $u=e^{y}$ in 
(25), frustration function can be alternatively expressed in the following form

$$
\operatorname{Fr}(x ; 0, \sigma)=\int_{-\infty}^{+\infty} \frac{1}{\sqrt{2 \pi \sigma^{2}}} \exp \left(-x e^{2 y}\right) e^{\frac{-\left(y+\sigma^{2}\right)^{2}}{2 \sigma^{2}}} d y .
$$

The first derivative of frustration function can be obtained as

$$
\frac{d \operatorname{Fr}(x ; 0, \sigma)}{d x}=\int_{-\infty}^{+\infty} \frac{-\exp \left(-x e^{2 y+4 \sigma^{2}}\right)}{\sqrt{2 \pi \sigma^{2}}} e^{\frac{-\left(y+\sigma^{2}\right)^{2}}{2 \sigma^{2}}} d y
$$

which is simply equal to

$$
\frac{d \operatorname{Fr}(x ; 0, \sigma)}{d x}=-\operatorname{Fr}\left(e^{4 \sigma^{2}} x ; 0, \sigma\right) .
$$

Using the chain rule, we can therefore conclude that

$$
\frac{d \operatorname{Fr}\left(f\left(K_{T}\right) ; 0, \sigma\right)}{d K_{T}}=-f^{\prime}\left(K_{T}\right) \operatorname{Fr}\left(e^{4 \sigma^{2}} f\left(K_{T}\right) ; 0, \sigma\right) .
$$

Noting $f^{\prime \prime}\left(K_{T}\right)=0$, we obtain

$$
\frac{d^{2} \operatorname{Fr}\left(f\left(K_{T}\right) ; 0, \sigma\right)}{d K_{T}^{2}}=e^{4 \sigma^{2}}\left(f^{\prime}\left(K_{T}\right)\right)^{2} \operatorname{Fr}\left(e^{8 \sigma^{2}} f\left(K_{T}\right) ; 0, \sigma\right) .
$$

Therefore, the second derivative of the PEP expression of Protocol III is positive which leads to the convexity of PEP expression and accordingly to the convexity of the objective function $\Gamma_{\text {III }}$ given by (46) under the considered assumption of $\beta<<1$.

The simplified PEP expressions under the assumption of $\beta<<1$ for Protocols I and II are given by (31) and (35). These are in the form of product of two frustration functions (i.e., $\left.G=\operatorname{Fr}\left(f_{1}\left(K_{T}\right) ; 0, \sigma\right) \operatorname{Fr}\left(f_{2}\left(K_{T}\right) ; 0, \sigma^{\prime}\right)\right)$ and are again a function of only the optimization parameter $K_{T}$. Their second derivatives are obtained as

$$
\begin{aligned}
\frac{d^{2} G}{d K_{T}^{2}}= & \operatorname{Fr}^{\prime \prime}\left(f_{1} ; 0, \sigma\right) \operatorname{Fr}\left(f_{2} ; 0, \sigma^{\prime}\right)+\operatorname{Fr}\left(f_{1} ; 0, \sigma\right) \operatorname{Fr}^{\prime \prime}\left(f_{2} ; 0, \sigma^{\prime}\right) \\
& +2 \operatorname{Fr}^{\prime}\left(f_{1}\right) \operatorname{Fr}^{\prime}\left(f_{2} ; 0, \sigma^{\prime}\right) .
\end{aligned}
$$

The first and second terms in the right hand side of (60) are positive for all $K_{T}$ noting that frustration function and its second derivative are positive as the latter is shown by (59). The third term is also positive since $f_{1}^{\prime}\left(K_{T}\right)$ and $f_{1}^{\prime}\left(K_{T}\right)$ are of the same sign for Protocols I and II assuming $\beta<<1$. Therefore we conclude that the second derivative of PEP expressions given by (31) and (35) are positive. Since the objective functions $\Gamma_{I}$ and $\Gamma_{I I}$ given by (44) and (45) are sum of PEP expressions, their second derivatives are also positive indicating their convexity under the assumed scenario of $\beta<<1$.

\section{APPENDIX II}

\section{INFINITE DIVERSITY ORDER FOR LOG-NORMAL FADING} CHANNELS

Lemma: Let $Y$ be a log-normal random variable with parameters $\left(-\sigma^{2}, \sigma\right)$ and cumulative distribution function $F(y)$. Let $y_{0}$ and $s$ be positive real numbers, Then the frustration function $\operatorname{Fr}(s ; 0, \sigma)$ can be bounded as

$$
\operatorname{Fr}(s ; 0, \sigma)<\frac{1}{F\left(y_{0}\right)} \int_{0}^{y_{0}} e^{-s y^{2}} d F(y) .
$$

Proof of lemma: Noting that $\exp \left(-s y^{2}\right)$ is always less than 1 and is a decreasing function, we can write the following inequalities

$$
\int_{0}^{y_{0}} e^{-s y^{2}} d F(y)>e^{-s y_{0}^{2}} \int_{0}^{y_{0}} d F(y)=e^{-s y_{0}^{2}} F\left(y_{0}\right),
$$

$$
\int_{y_{0}}^{\infty} e^{-s y^{2}} d F(y)<e^{-s y_{0}^{2}} \int_{y_{0}}^{\infty} d F(y)=e^{-s y_{0}^{2}}\left[1-F\left(y_{0}\right)\right] .
$$

Using (62) and (63), we have

$$
\int_{y_{0}}^{\infty} e^{-s y^{2}} d F(y)<\frac{\left[1-F\left(y_{0}\right)\right]}{F\left(y_{0}\right)} \int_{0}^{y_{0}} e^{-s y^{2}} d F(y)
$$

which can be simplified as

$$
\int_{0}^{\infty} e^{-s y^{2}} d F(y)<\frac{1}{F\left(y_{0}\right)} \int_{0}^{y_{0}} e^{-s y^{2}} d F(y) .
$$

Note that frustration function can be also represented as

$$
\begin{aligned}
\operatorname{Fr}(s ; 0, \sigma) & =\int_{0}^{\infty} \frac{\exp \left(-s y^{2}\right)}{y \sqrt{2 \pi \sigma^{2}}} e^{\frac{-\left(\ln y+\sigma^{2}\right)^{2}}{2 \sigma^{2}}} d y \\
& =\int_{0}^{\infty} e^{-s y^{2}} d F(y) .
\end{aligned}
$$

Inserting (66) into (65), the proof of lemma is completed.

Theorem: The error rate performance of direct transmission in log-normal fading channel has the diversity order of infinity $(d=\infty)$.

Proof: Consider (52) and replace $\sigma_{S D} / \xi$ with $\sigma$ and $S N R$ with $s$ for notational convenience. Then using (66), the error rate performance of direct transmission is bounded by

$$
P_{b} \leq \frac{1}{2} \operatorname{Fr}(s ; 0, \sigma)=\frac{1}{2} \int_{0}^{\infty} e^{-s x^{2}} d F(x)
$$

where $F(x)$ is the cumulative distribution function of lognormal random variable $X$ with parameters $\left(-\sigma^{2}, \sigma\right)$. Using the lemma, (67) can be bounded by

$$
P_{b} \leq \frac{1}{2} \operatorname{Fr}(s ; 0, \sigma)<\frac{1}{2 F\left(y_{0}\right)} \int_{0}^{y_{0}} \frac{e^{-s x^{2}}}{x \sqrt{2 \pi \sigma^{2}}} e^{\frac{-\left(\ln x+\sigma^{2}\right)^{2}}{2 \sigma^{2}}} d x .
$$

Substituting $y=s x^{2}$ in the integral, we obtain

$$
P_{b}<\frac{1}{2 F\left(y_{0}\right)} \int_{0}^{y_{0}} \frac{e^{-y}}{y \sqrt{8 \pi \sigma^{2}}} e^{\frac{-\left(\ln y-\ln s+2 \sigma^{2}\right)^{2}}{8 \sigma^{2}}} d y
$$

which can be further simplified as

$$
P_{b}<\frac{e^{\ln s\left(4 \sigma^{2}-\ln s\right)}}{2 F\left(y_{0}\right)} \int_{0}^{y_{0}} \frac{e^{-y} e^{2 \ln s \ln y}}{y \sqrt{8 \pi \sigma^{2}}} e^{\frac{-\left(\ln y+2 \sigma^{2}\right)^{2}}{8 \sigma^{2}}} d y .
$$

Since $\exp \left(2 \ln s \ln y_{0}\right) \geq \exp (2 \ln s \ln y), \forall y \in\left[0, y_{0}\right]$, we can further simplify (70) as

$$
\begin{aligned}
P_{b} & <\frac{\exp \left(\ln s\left(2 \ln y_{0}+4 \sigma^{2}-\ln s\right)\right)}{2 F\left(y_{0}\right)} \int_{0}^{y_{0}} \frac{e^{-y}}{y \sqrt{8 \pi \sigma^{2}}} e^{\frac{-\left(\ln y+2 \sigma^{2}\right)^{2}}{8 \sigma^{2}}} d y \\
& <\frac{\exp \left(\ln s\left(2 \ln y_{0}+4 \sigma^{2}-\ln s\right)\right)}{2 F\left(y_{0}\right)} .
\end{aligned}
$$


The second inequality in (71) holds since the integral is bounded by the expectation of $\exp (-y)$ with respect to a lognormal random variable and this expectation is less than one, noting $\exp (-y)<1, \forall y>0$. From (71), we can readily obtain

$$
-\frac{\log P_{b}}{\log s}=-\frac{\ln P_{b}}{\ln s}>\ln s-2 \ln y_{0}-4 \sigma^{2}+\frac{\ln 2 F\left(y_{0}\right)}{\ln s} .
$$

Taking limit of both sides of the inequality in (72) as $s \rightarrow \infty$, we have

$$
\begin{aligned}
d & =\lim _{s \rightarrow \infty}-\frac{\log P_{b}}{\log s} \\
& \geq \limsup _{s \rightarrow \infty}\left[\ln s-2 \ln y_{0}-4 \sigma^{2}+\frac{\ln 2 F\left(y_{0}\right)}{\ln s}\right]=\infty .
\end{aligned}
$$

Thus the proof is completed.

\section{REFERENCES}

[1] V. Tarokh, N. Seshadri, and A. R. Calderbank, "Space-time codes for high data rate wireless communication: performance analysis and code construction," IEEE Trans. Inform. Theory, vol. 44, pp. 744-765, Mar. 1998.

[2] S. M. Alamouti, "A simple transmit diversity technique for wireless communications," IEEE J. Select. Areas Commun., vol. 16, pp. 14511458, Oct. 1998.

[3] V. Tarokh, H. J. Jafarkhani, and A. R. Calderbank, "Space-time block codes from orthogonal designs," IEEE Trans. Inform. Theory, vol. 45, pp. 1456-1467, July 1999.

[4] A. Sendonaris, E. Erkip, and B. Aazhang, "User cooperation diversitypart I: system description,” IEEE Trans. Commun., vol. 51, pp. 19271938, Nov. 2003.

[5] A. Sendonaris, E. Erkip, and B. Aazhang, "User cooperation diversitypart II: implementation, aspects and performance analysis," IEEE Trans. Commun., vol. 51, pp. 1939-1948, Nov. 2003.

[6] J. N. Laneman and G. W. Wornell, "Distributed space-time-coded protocols for exploiting cooperative diversity in wireless networks," IEEE Trans. Inform. Theory, vol. 49, no. 10, pp. 2451-2425, Oct. 2003.

[7] J. N. Laneman, D. N. C. Tse, and G. W. Wornell, "Cooperative diversity in wireless networks: efficient protocols and outage behavior," IEEE Trans. Inform. Theory, vol. 50, no. 12, pp. 3062-3080, Dec. 2004.

[8] R. Nabar, H. Bolcskei, and F. Kneubuhler, "Fading relay channel: performance limits and space-time signal design," IEEE J. Select. Areas Commun., vol. 22, pp. 1099-1109, Aug. 2004.

[9] A. Nosratinia, T. Hunter, and A. Hedayat, "Cooperative communication in wireless networks," IEEE Commun. Mag., vol. 42, no. 10, pp. 68-73, Oct. 2004

[10] K. Azarian, H. E. Gamal, and P. Schniter, "On the achievable diversitymultiplexing tradeoff in half duplex cooperative channels," IEEE Trans. Inform. Theory, vol. 51, no. 12, pp. 4152-4172, Dec. 2005.

[11] S. Y. Seidel, K. Takamizawa, and T. S. Rappaport, "Application of second-order statistics for an indoor radio channel model," in Proc. IEEE Veh. Technol. Conf., San Fransisco, CA, May 1989, pp. 888-892.

[12] F. Lotse, J. E. Berg, and R. Bownds, "Indoor propagation measurement at $900 \mathrm{MHz}$," in Proc. IEEE Veh.Technol. Conf., Denver, CO, May 1992, pp. 629-632.

[13] R. Ganesh and K. Pahlavan, "Statistical modeling and computer simulation of indoor radio channel," in Proc. IEE Communications, Speech and Vision, vol. 138, no. 3, pp. 153-161, June 1991.

[14] H. Hashemi, "The indoor radio propagation channel," Proc. IEEE, vol. 81, pp. 943-967, July 1993

[15] M. S. Alouini and M. K. Simon, "Dual diversity over correlated lognormal fading channels," IEEE Trans. Commun., vol. 50, pp. 1946-1959, Dec. 2002.

[16] T. Piboongungon and V. A. Aalo, "Outage probability of L-branch selection combining in correlated lognormal fading channels," Electron. Lett., vol. 40, no. 14, pp. 263, July 2004.

[17] A. Papoulis, Probability, Random Variables, and Stochastic Processes. New York: McGraw-Hill, 1991.
[18] J. W. Mark and W. Zhuang, Wireless Communications and Networking. Pearson Education, Inc., 2003.

[19] H. Ochiai, P. Mitran, and V. Tarokh, "Design and analysis of collaborative diversity Protocols for wireless sensor networks," in Proc. IEEE Veh. Technol. Conf., vol. 7, pp. 4645-4649, Fall 2004.

[20] S. J. Halme, B. K. Levitt, and R. S. Orr., "Bounds and approximations for some integral expression involving lognormal statistics," MIT Res. Lab. Electron. Quart. Prog. Rept., 1969.

[21] A. Host-Madsen and J. Zhang, "Capacity bounds and power allocation in wireless relay channel," IEEE Trans. Inform. Theory, vol. 51, pp. 2020-2040, June 2005.

[22] N. Ahmed and B. Aazhang, "Outage minimization with limited feedback for the fading relay channel," IEEE Trans. Commun., vol. 54, pp. 659559, Apr. 2006.

[23] M. O. Hasna and M. S. Alouini, "Optimum power allocation for relayed transmissions over Rayleigh-fading channels," IEEE Trans. Wireless Commun., vol. 3, pp. 1999-2004, Nov. 2004.

[24] Y. Jing and B. Hassibi, "Distributed space-time coding in wireless relay networks," IEEE Trans. Wireless Commun., vol. 5, pp. 3524-3536, Dec. 2006.

[25] X. Deng and A. M. Haimovich, "Power allocation for cooperative relaying in wireless networks," IEEE Commun. Lett., vol. 9, pp. 994996, Nov. 2005.

[26] M. M. Fareed and M. Uysal, "BER-optimized power allocation for fading relay channels," accepted for publication in IEEE Trans. Wireless Commun.

[27] J. G. Proakis, Digital Communications, 4th edition. New York: McGrawHill, Inc., 2001.

[28] Optimization Toolbox, Documentation, The MathWorks, "Optimization toolbox 3 user's guide," Sept. 2007, http:// www.mathworks.com/access/helpdesk/help/pdf_doc/optim/optim_tb.pdf.

[29] R. Narasimhan, "Finite-SNR diversity-multiplexing tradeoff for correlated Rayleigh and Rician MIMO channels," IEEE Trans. Inform. Theory, vol. 52, pp. 3965-3979, Sept. 2006.

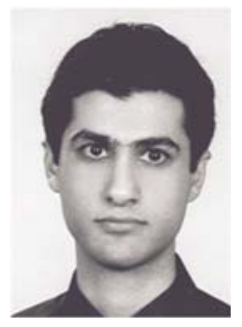

Majid Safari received B.Sc. in Electrical Engineering from the University of Tehran, Tehran, Iran, in 2003 and M.S. in Electrical Engineering from Sharif University of Technology, Tehran, Iran, in 2005. He is currently a Ph.D. student in Electrical Engineering, University of Waterloo, Canada. His research interests include cooperative communications, free-space optical communications, and optical fiber communications.

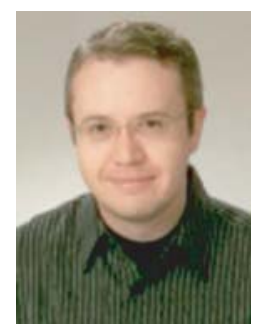

Murat Uysal was born in Istanbul, Turkey in 1973. $\mathrm{He}$ received the B.Sc. and the M.Sc. degree in electronics and communication engineering from Istanbul Technical University, Istanbul, Turkey, in 1995 and 1998, respectively, and the Ph.D. degree in electrical engineering from Texas A\&M University, College Station, Texas, in 2001. Since 2002, he has been with the Department of Electrical and Computer Engineering, University of Waterloo, Canada, where he is now an Associate Professor. His general research interests lie in communications theory and signal processing for communications with special emphasis on wireless applications. Specific research areas include space-time coding, MIMO techniques, performance analysis over fading channels, and cooperative communications.

Dr. Uysal is an Associate Editor for IEEE Transactions on Wireless Communications and IEEE Communications Letters. He also served as a Guest Co-Editor for Wiley Journal on Wireless Communications and Mobile Computing's Special Issue on "MIMO Communications" published in 2004. Over the years, he has served on the technical program committee of more than 50 international conferences in the communications area. He recently co-chaired IEEE ICC'07 Communication Theory Symposium. He is currently chairing Communications and Networking Symposium within the Canadian Conference on Electrical and Computer Engineering. Dr. Uysal is a Senior IEEE member. 